\title{
Visualization Location in Q235B Steel Structure by Using the Acoustic Emission Tomography
}

\author{
Yu Jiang1*, Zhong Yang ${ }^{1}$, Feiyun $\mathrm{Xu}^{2}$, Bingsheng $\mathrm{Xu}^{3}$ and Antolino Gallego ${ }^{4}$ \\ 1. College of Intelligent Science and control Engineering, Jinling Institutes of Technology, Nanjing, 211169, China \\ 2. School of Mechanical Engineering, Southeast University, Nanjing, 211189, China \\ 3. School of Science, Nanjing Tech University, Nanjing, 211800, China \\ 4. School of Building Engineering, Campus Fuentenueva, University of Granada, Granada, 18071, Spain
}

\begin{abstract}
AET (acoustic emission tomography) is a new visualization location method for inspecting and locating the internal damages in the structures. In this paper, the AET is applied to detect and visualize damage source locations in the Q235B steel structure to validate its effectiveness. Firstly, the ABAQUS/Explicit simulation model of the steel structure with one damaged circle in its center is built. And then, the simulated AE signals are obtained to establish the AE tomography. The results show that the damaged circle in the created model can be visualized clearly with the AET in its original location. Finally, the damage source location based on AET is compared with the traditional TOA (time of arrival) location method, and the better location accuracy is obtained. And this fact that further illustrates the AET has a great potential in the application of structure damage detection.
\end{abstract}

Key words: AET, damage location, visualization.

\section{Introduction}

$\mathrm{AE}$ (acoustic emission) is a transient wave resulting from the sudden release of stored energy during a damage process [1]. It can be used to identify the evolution of damage in structures as a powerful nondestructive testing method. In civil engineering, the complexity of material and different forms of components make the received AE signals very intricate, which have been challenging in damage identification. Classical method is that $\mathrm{AE}$ parameters are recorded and analyzed to identify damages. With advanced in computing power, waveform of AE signals is analyzed as a diagnostic tool. Although both two methods are applied nowadays with some success for diverse applications, to better diagnose and identify early internal damage of structures, the visualization of the damaged location from the $\mathrm{AE}$ inspection is required.

Tomography is an emerging technique for nondestructive evaluation, which aims to visualize the

Corresponding author: Yu Jiang, teacher, Dr. degree, research fields: acoustic emission detect, information of things. internal structure of various types of objects by using two- or three-dimensional representation, so that better identification of anomalous regions and determination of physical properties of the measured region can be achieved. In terms of in situ application, Traditional tomography methods such as Computer Tomography, Travel time Tomography and Ultra-acoustic tomography fail to meet the need of more global and local property information distributions throughout object structures. AET is a new visualization method for inspecting and locating the internal damages in the structures, which is based on the time-travel tomography with AE events as its signal sources.

\section{AET Theory}

$\mathrm{AE}$ tomography uses the AE events as signal sources for the travel-time tomography. It contains source location algorithm and travel tomography algorithm. This technique represents an important improvement on traditional AE data analysis. It not only leads to a new imaging technique that the $\mathrm{AE}$ sources positions in the specimen are visualized in terms of a locally 
varying wave speed distribution, but also leads to significantly better localization of AE events. See for Eq. (1) the arrival time of the AE signal received at each sensor can be stated as:

$$
T_{k}^{A}=T_{k}^{0}+\sum w_{i j}^{k} s_{i j}
$$

where, $k$ refers to each ray from the acoustic event source to each sensor $k=1,2, \ldots r,(i, j)$ is index indicating the position of each tomographic cell $i=1$, $2, \ldots, m, j=1,2, \ldots, n . \quad s_{i j}=1 / C_{i j}$ is the "slowness" of the signal propagation along the tomographic cell, defined as the reciprocal of its propagation speed $c_{i j} . w_{i j}$ represents the distance travelled by the kth ray inside the cell $(i, j)$. Note that most of these elements are zero, since only a relative small number of cells are crossed by each ray. $T_{k}^{A}$ is the time of measured arrival of the signal from ray $\mathrm{k}$ at the corresponding sensor. $T_{k}^{0}$ is the time of measured occurrence of the event that originates the ray $k$. The solution of Eq. (1) can be obtained iteratively with a process known as ART (algebraic reconstruction technique) [2].

\section{Numerical Simulation}

In order to address the theory of the AET, the ordinary Q235B steel plate (China Code) is chosen as

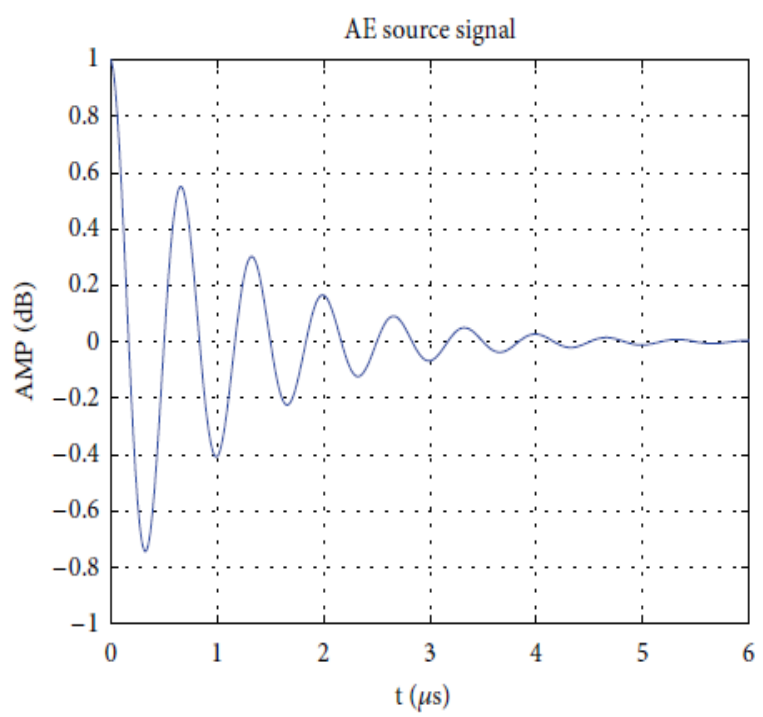

(a) AE source signal the main material of the sample in the numerical simulation process, which is a typical homogeneous media. The model of steel structure is established using commercial package ABAQUS/Explicit [3-6], as shown in Fig. 1 and the main parameters of the steel plate (Q235B) structure model are shown in Ref. [7]. For each excited AE source event, time-domain waveform of $\mathrm{AE}$ source signal is shown in Fig. 2a. Point $A$ is the occurrence time of signal arrival and point $B$ is the peek time in the simulated time-domain waveform (Fig. 2b).

\section{Results and Data Analysis}

The simulation process was repeated for the complete data set, a total of more than one hundred $\mathrm{AE}$ events were excited randomly along the circumference of the damaged circle inside the FE model. Afterwards, the peak-arrival time of the time-domain signals

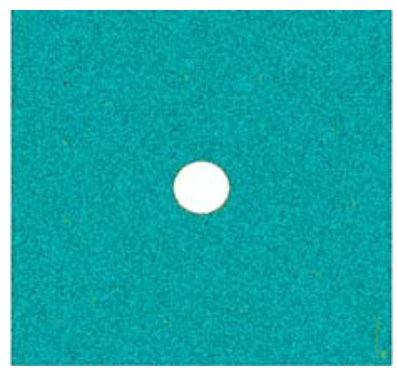

Fig. 1 AET 2D simulation model.

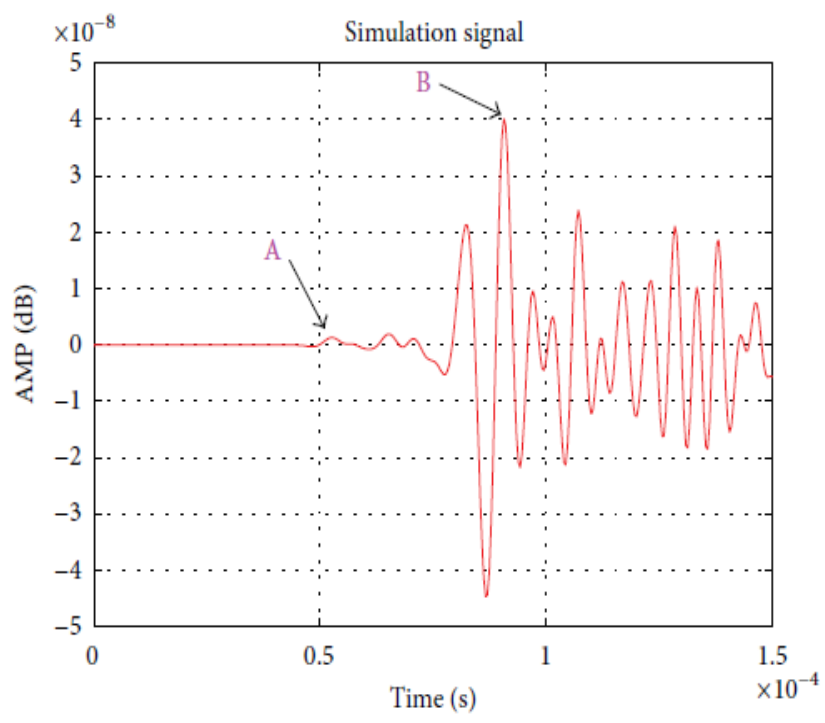

(b) Simulation signals (FE)

Fig. 2 Simulation AE source and AE signal. 


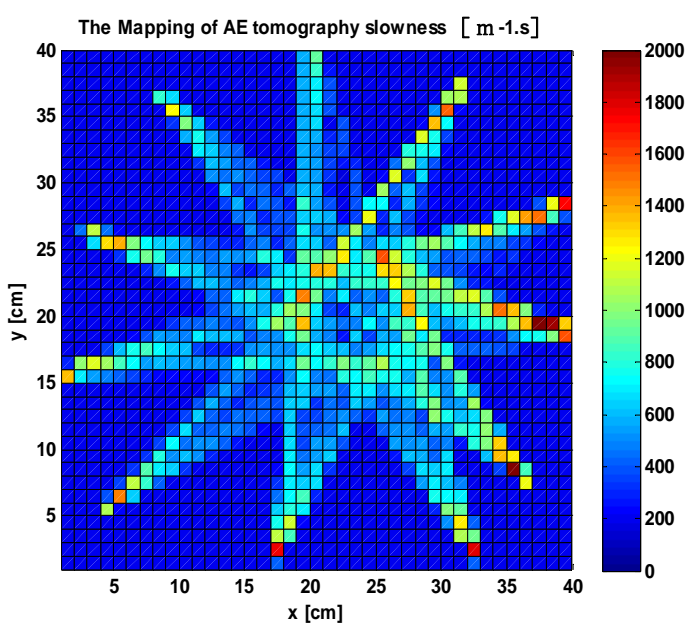

(a) 36AEevents

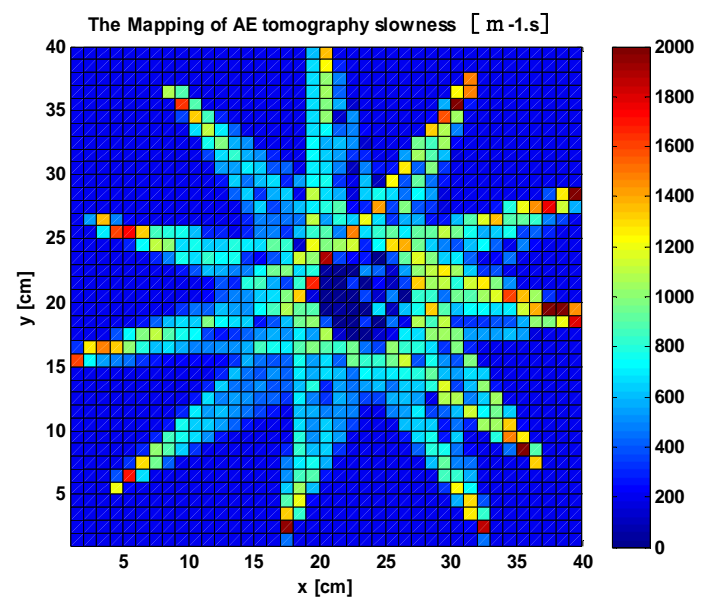

(c) 108AEevents

Fig. 3 AET results.

received at each sensor was determined by using manual picking waveform ( $\mathrm{P}$ wave) data at the point $\mathrm{B}$. Arrival-time difference series computed were served as input for the AE tomography algorithm.

As shown in Fig. 3, it can be seen that a damaged circle location in the FE model of Q235B steel structure is located and visualized by the $\mathrm{AE}$ tomography algorithm. As the number of AE events is increasing, each new $\mathrm{AE}$ event can lead to a better approximation in spatial velocity field. Wave speed changes in the defective region become more obvious than the other area in the model. Figs. 3a-3d display the results from the different $\mathrm{AE}$ events in the mapping of the AE tomography constructed and these results show

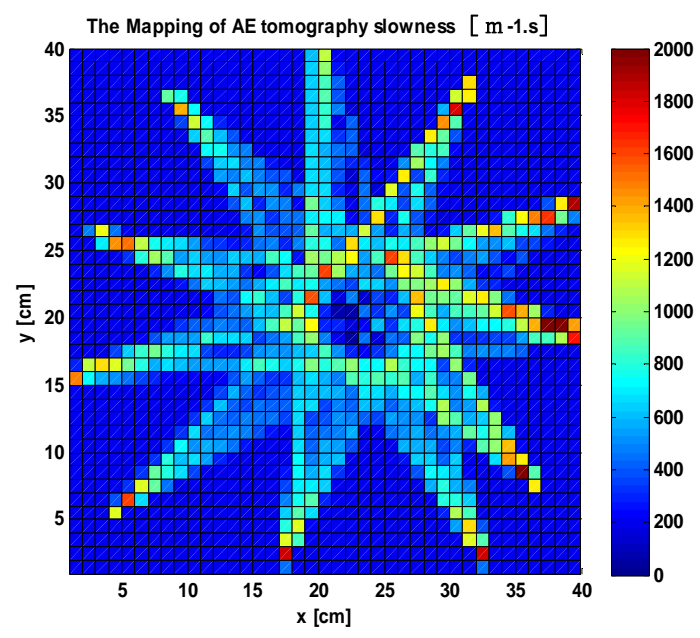

(b) $72 \mathrm{AE}$ events

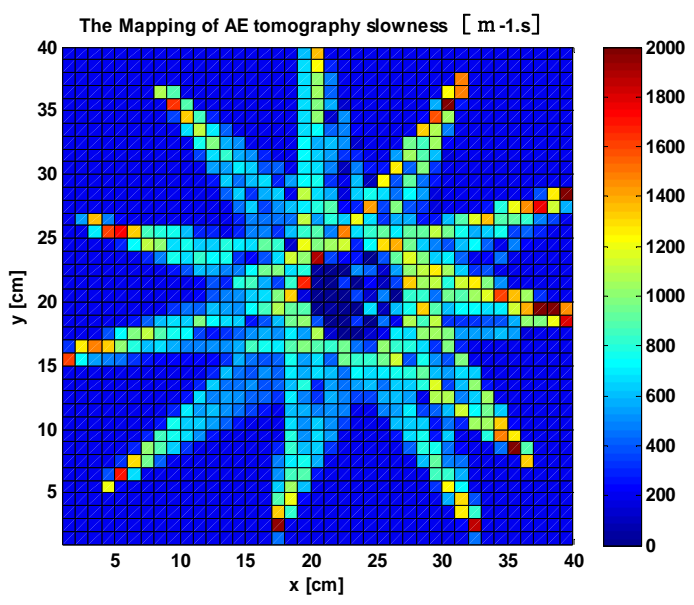

(d) 180AE events

a remarkable improvement in the $\mathrm{AE}$ tomographic image quality. More than one hundred of AE events, especially, such as 180AE events, are computed, the damaged circle position becomes pretty clear, and local damaged area slowness value arrives at the maximum in the tomographic image (Fig. 3c). A further increase of the number of $\mathrm{AE}$ events intends to improve the quality of the images automatically, but the results are not prominent (Fig. 3d). This result shows that the ray coverage of the tomography cells reaches a certain level, and a saturation behavior has occurred.

\section{Compared with TOA Algorithm}

Seen from Fig. 3 some artifacts in the tomographic 
Table 1 One hundred and eighty source location errors.

\begin{tabular}{lll}
\hline Algorithms & ART & TOA \\
\hline ME & 7.330 & 7.599 \\
MR & 0.072 & 0.198 \\
WAE & 0.203 & 0.217 \\
SD & 1.060 & 1.124 \\
WASD & 0.192 & 0.248 \\
\hline
\end{tabular}

images are also observed, and it is most likely caused by the simple straight line approximation of the $\mathrm{AE}$ tomography model. The nonuniform ray converges of the model leading to large differences in the number of rays passing through the equally sized tomography cells may be another reason. However, when a saturation behavior of the tomography images is obtained, image quality changes have a little effect on the precision of $\mathrm{AE}$ source location to some extent. Since the locations of all AE events in the simulation and the experiment are prior knowledge, the accuracy of AE source location is further studied. One hundred and eighty AE (180 AE) events are chosen to evaluate source location accuracy in traditional TOA location algorithm and $\mathrm{AE}$ tomography location algorithm, respectively. And ME (mean error), MR (mean residual), WAE (weight average error), SD (standard deviation), and WASD (weight average standard deviation) are considered to be the indexes to evaluate source location error [10-12]. The error results are shown in Table 1.

As shown by Table 1, simulation results are better. Traditional TOA error is bigger than AE tomography source location. This fact that further illustrates the AET has a great potential in the application of structure damage detection.

\section{Conclusions}

AE tomography methodology (ART) is proposed and implemented in AE simulation test on Q235B steel specimen with one hole (defect). The results show that the new proposed method can reveal the details of internal structure of the specimen. One damaged source location can be successful visualized with AET algorithm. This technique would be considered as a great potential for future engineering applications in AE fields. And the following conclusions have been reached.

The finite element simulation (ABAQUS/Explicit) model of Q235B steel structure is built. Simulated AE source is excited to produce AE signal and numerical data set is obtained to serve as input of AE tomography algorithm. Good simulation results are obtained.

Compared with the results between AE tomography location and traditional TOA location, the experimental results obtain good results from the point of source location error such as mean error and mean residual, etc;

A considerable improvement over conventional TOA location algorithm is obtained by using the AE tomography location algorithm.

\section{Acknowledgments}

This work is supported by the National Natural Science Foundation of China (No.51575101, No.51305176), the introduction fund of High-level talents of JIT (No.JIT6201623). In addition, the author is grateful to the EGWAE reviewers for their future reviewed work.

\section{References}

[1] Kak, A. C., and Slaney, M. 1988. "Principles of Computerized Tomographic Imaging.” Medical Physics 29 (1).

[2] Jiang, Y., Xu, F., and Xu, B. 2015. “Acoustic Emission Tomography Based on Simultaneous Algebraic Reconstruction Technique to Visualize Damage Source Location in Q235B Steel Plate.” Mechanical System and Signal Processing 65: 452-64.

[3] Jiang, Y., Xu, F., and Xu, B. 2015. "Simulation and Experimental Investigation on the AE Tomography to Improve AE Source Location in the Concrete Structure.” Mathematical Problems in Engineering 2014 (5): 1-10.

[4] Ito, F., Nakahara, F., and Kawano, R. 2001. "Visualization of Failure in a Pull-out Test of Cable Bolts Using X-ray CT.” Construction and Building Materials 15: 263-70.

[5] Fan, S., Zhao, S., Zhang, Q., and Yongyi, L. I. 2013. "Plasticmechanism of Multi-pass Double-Roller Clamping Spinning for Arc-shaped Surface Flang.” Chinese Journal of Mechanical Engineering 26: 1127-37.

[6] ABAQUS, Inc. 2008. “ABAQUS Analysis User’s Manual 
Version 6.8”.

[7] Baxter, M. G., Pullin, R., and Holford, K. M. 2007. "Detla T. Source Location for Acoustic Emission.” Mech. Syst. Signal Process 21: 1512-20.

[8] Maji, A. K., and Satpathi, D. 1995. "Acoustic Emisssion Source Location Based on Lamb Waves.” In Proceedings of Engineering Mechanics, 1: 597-600.

[9] Yadav, S., Gu, A., and Luo, Y. 2015. “Continuous Condition Monitoring of Reinforced Concrete Using an Active Diagnosis Method.” Structural Health Monitoring
1: $1-9$.

[10] Schubert, F. 2004. "Basic Principles of Acoustic Emission Tomography.” In 26th European Conference on Acoustic Emission Testing, 693-708.

[11] Schubert, F. 2006. “Tomography Techniques for Acoustic Emission Monitoring.” In 28th European Conference on NDT-September, Berlin (Germany)-ECNDT.

[12] Aggelis, D. G. 2011. "Damage Characterisation of Inhomogeneous Materials: Experiments and Numerical Simulations of Wavepropagation.” Strain 47: 525-33. 